\title{
Expression of bladder cancer-associated glycans in murine tumor cell lines
}

\author{
MARINA ALBERTÓ $^{1}$, HECTOR ADRIÁN CUELLO ${ }^{1}$, CYNTHIA ANABELLA GULINO $^{1}$, MARINA PIFANO ${ }^{1}$, \\ DENISE BELGOROSKY ${ }^{2}$, MARIANO ROLANDO GABRI ${ }^{1}$, ANA MARÍA EIJÁN ${ }^{2}$ and VALERIA INÉS SEGATORI ${ }^{1}$ \\ ${ }^{1}$ Laboratory of Molecular Oncology, Quilmes National University, Bernal B1876BXD; \\ ${ }^{2}$ Research Area, Instituto de Oncología Angel H. Roffo, Universidad de Buenos Aires, Buenos Aires 1417 DTB, Argentina
}

Received July 16, 2018; Accepted December 17, 2018

DOI: $10.3892 / \mathrm{ol} .2019 .9995$

\begin{abstract}
The characterization of murine cell lines is of great importance in order to identify preclinical models that could resemble human diseases. Aberrant glycosylation includes the loss, excessive or novel expression of glycans and the appearance of truncated structures. MB49 and MB49-I are currently the only two murine cell lines available for the development of preclinical bladder cancer models. The glycans Lewis X (LeX), Sialyl lewis X (SLeX) and Sialyl Tn (STn) have previously been associated with aggressiveness, dissemination and poor prognosis in human bladder cancer, additionally $\mathrm{N}$-glycolyl GM3 (NGcGM3) is a neo-antigen expressed in many types of tumors; however, to the best of our knowledge, its expression has not previously been assessed in this type of cancer. Taking into account the relevance of glycans in tumor biology and considering that they can act as targets of therapies and biomarkers, the present study evaluated the expression of LeX, SLeX, STn and NGcGM3 in MB49 and MB49-I cells, in different growth conditions such as monolayer cultures, three-dimensional multicellular spheroids and mouse heterotopic and orthotopic tumors. The expression of LeX was not detected in either cell line, whereas SLeX was expressed in monolayers, spheroids and orthotopic tumors of both cell lines. STn was only identified in MB49 monolayers and spheroids. There are no reports concerning the expression of NGcGM3 in human or murine bladder cancer. In our hands, MB49 and MB49-I expressed this ganglioside in all the growth conditions evaluated. The assessment of its expression in cancer cell lines and patient tumors is of great importance, considering the relevance of this ganglioside in tumor biology. The data obtained by the present study demonstrates that glycan expression may be substantially altered depending on the growth conditions, highlighting the importance of the
\end{abstract}

Correspondence to: Dr Valeria Inés Segatori, Laboratory of Molecular Oncology, Quilmes National University, 352 Roque Saenz Peña, Bernal B1876BXD, Argentina

E-mail: valeria.segatori@unq.edu.ar

Key words: bladder cancer, glycans, murine models, MB49, MB49-I characterization of murine cancer models. To the best of our knowledge, the present study is the first to examine the expression of cancer-associated glycans, in the two murine cell lines available for the development of preclinical studies in bladder cancer.

\section{Introduction}

Bladder cancer accounts for approximately 165,000 deaths every year and is rated as the eleventh most common malignancy worldwide (1). Urothelial carcinomas, which comprise $90 \%$ of bladder tumors, are generally classified into three states: non-muscle-invasive, muscle-invasive, or metastatic (2) with a 5-year survival rates of less than $15 \%$ for those patients with metastatic disease (3). Depending on the stage of the tumor, treatment for bladder cancer consists in combinations of surgery, radiotherapy, chemotherapy, intravesical administration of BCG and, most recently, immune checkpoint inhibitors (4-9).

Cancer research has the constant challenge of developing preclinical models that recreate human disease allowing the evaluation and validation of anticancer therapies. The use of murine tumor cell lines and the development of tumors in immunocompetent mice appear as useful models for studies involving the microenvironment and the immune system, among others. In the particular case of bladder cancer, mouse models include carcinogen-induced tumors and orthotopic and heterotopic tumors developed by murine bladder cancer cell lines (10-12). There are two widely used murine tumor cell lines: MB49, developed from a 7,12-dimethylenzanthacene-induced bladder tumor in C57BL/6 mouse and MBT-2, developed from an FANFT-induced bladder tumor in $\mathrm{C} 3 \mathrm{H} / \mathrm{He}$ mouse $(13,14)$. The latter was found to be contaminated with replicating type $\mathrm{C}$ retrovirus, turning this cell line in an unreliable model for immune assays $(15,16)$. This leaves MB49 as virtually the only murine bladder cancer cell line used nowadays $(11,12,14)$. Lodillinsky et al (17), generated a new bladder cancer cell line (MB49-I) by successive in vivo passages of primary tumor obtained by inoculating MB49 in C57BL/6 mice. MB49-I exhibited more invasive properties and its orthotopic inoculation generated invasive tumors similar to invasive human bladder cancer, therefore making it an extremely interesting preclinical model. These two murine bladder cancer models 
have been characterized in various aspects (17-19); however, there is no information regarding their glycan expression profile.

Aberrant glycosylation is a phenomenon described in the malignant transformation and includes loss or excessive expression of certain glycans, appearance of incomplete or truncated structures and the appearance of novel glycans that can be associated to proteins or lipids $(20,21)$. Changes in cellular glycoproteins and glycolipids have been proposed as a new cancer hallmark due to their association with malignant transformation and cancer progression (21). These glycoconjugates are involved in many biological processes and have a key role in several steps of tumor development and progression such as cell-cell adhesion, cell-matrix interaction, inter and intracellular signaling, immune surveillance and many others (22). Taking into account that these glycans are differentially expressed in cancer over normal cells, they have been used as cancer biomarkers $(23,24)$ and have been the target of numerous therapies for cancer treatment, including monoclonal antibodies against glycans, vaccines and glycan-directed CAR-T cells, among others (25-29).

Several glycans have been studied in human bladder cancer, among them the blood group antigen Lewis X (LeX) has been extensively validated as an urothelial carcinoma biomarker, as it can be detected in exfoliated urothelial cells (30-32). This glycan is expressed in bladder tumors regardless of its grade or stage; but is not commonly present in urothelial cells $(31,32)$. Furthermore, it has been associated with invasive and metastatic potential in this type of cancer $(30,33)$. The sialylated variant of LeX, Sialyl Lewis X (SLeX), is also frequently expressed in tumor samples and human bladder cancer cell lines. This glycan has a key role in the recognition of selectins and it is involved in tumor cells dissemination. In particular, Fujii et al (34) reported SLeX involvement in E-selectin-mediated adhesion of urothelial cancer cells to endothelium. In addition, the expression of SLeX in samples from bladder carcinoma patients was found to strongly correlate with invasive and metastatic clinical outcome (35). Another relevant glycan in bladder cancer is Sialyl Tn (STn). $\mathrm{STn}$ is a truncated aberrant O-glycan that tends to appear in carcinoma mucins (20). Several reports have demonstrated the expression of this antigen in high grade muscle-invasive bladder tumors and its correlation with aggressiveness, poor prognosis and disease dissemination (36-38). Despite STn association with bladder cancer malignancy, its presence has been associated with better response to BCG therapy due to the induction of a stronger inflammatory response mediated by macrophages (39). The ganglioside N-glycolyl GM3 (NGcGM3) is a tumor neoantigen which is expressed in several types of cancer (40-45), and has been validated as a target for the development of cancer immunotherapy. However, there are no publications regarding this glycan in urothelial carcinoma, making it an interesting potential target or biomarker in this indication.

The development of a preclinical model in oncology involves the use of a murine cancer cell line with the ability to generate tumors in mice and to recreate the disease described in humans. This includes the analysis of the expression of glycans relevant in bladder cancer, which are also targets of validated therapies in other indications (10-12). Glycan target-directed immunotherapies are a good example, since the validation of these therapies needs immunocompetent cancer mouse models, as the use of human cancer cell lines in immunosupressed mice is not suitable (26). MB49 and MB49-I are capable of developing tumors in syngeneic mice and possess biological characteristics similar to human bladder cancer (17), but there is no information regarding bladder cancer-associated glycans expression in these cell lines.

Taking into account the relevance of glycosylated targets in cancer therapy and the lack of information regarding this topic in murine bladder cancer models, the aim of this work was to characterize the expression of bladder cancer-associated glycans LeX, SLeX, STn, and NGcGM3 as part of the glycophenotype of most significant murine bladder cancer cell lines MB49 and MB49-I, including monolayer cultures, three-dimensional multicellular spheroids, and heterotopic and orthotopic tumors developed from these cell lines.

\section{Materials and methods}

Tumor cells and culture conditions. Murine bladder cancer cell line MB49 was obtained from Dr E.C. Lattime, Thomas Jefferson University, Philadelphia, USA. MB49-I was generated by Lodillinsky et al (17), as described previously. Human bladder cancer cell line T24 was purchased in ATCC (ATCC ${ }^{\circledR}$ HTB-4 $\left.{ }^{\mathrm{TM}}\right)$. Cell lines MB49, MB49-I and T24 were grown in RPMI medium (Gibco; Thermo Fisher Scientific, Inc., Waltham, MA, USA) supplemented with $10 \%$ heat-inactivated fetal bovine serum (FBS) (Gibco; Thermo Fisher Scientific, Inc.), $2 \mathrm{mM}$ glutamine and $80 \mu \mathrm{g} / \mathrm{ml}$ gentamicin at $37^{\circ} \mathrm{C}$ in $5 \% \mathrm{CO}_{2}$ atmosphere. Cell cultures were routinely subcultured twice a week by tripsinization using standard procedures.

Animals. Pathogen-free C57BL/6J mice approximately 10 weeks-old, with an average weight of $25 \mathrm{~g}$, were obtained from the Animal Care Division of UNLP (La Plata, Argentina). Up to 5 mice per cage were kept with water and food ad libitum in the animal house facility at Quilmes National University. All protocols were approved by the Institutional Committee for the Care and Use of Laboratory Animals at Quilmes National University.

Three-dimensional multicellular tumor spheroids growth. Spheroids were prepared using a modified protocol based on the liquid overlay technique described by Yuhas et al (46) Cell culture 96-well plates were underlayed with $1.5 \%$ agarose (GE Healthcare, Chicago, IL, USA) in RPMI-10\% FBS. Spheroids were initiated by inoculating 10,000 or 20,000 cells in RPMI-10\% FBS, followed by cycles of agitation. The medium was replaced once during the growth of the spheroids. At day 14 the spheroids were fixed in $4 \%$ paraformaldehyde, centrifuged and resuspended in $2 \%$ agarose. This material was embedded in paraffin and processed for histology.

Flow cytometry assay (FACS). Tumor cells were harvested with trypsin-EDTA solution, resuspended in serum-free RPMI, and $1 \times 10^{6}$ cells per sample were incubated with glycan's specific monoclonal antibodies (mAbs), biotinylated lectins, isotype control or PBS for $30 \mathrm{~min}$ at $4^{\circ} \mathrm{C}$ or room temperature according to the $\mathrm{mAb}$ datasheet. The $\mathrm{mAbs}$ used were: 
NeuGcGM3 (mouse anti-mouse 14F7, $20 \mu \mathrm{g} / \mathrm{ml}$; produced by the Center of Molecular Immunology, La Habana, Cuba); SLeX (mouse anti-mouse SNH3, $20 \mu \mathrm{g} / \mathrm{ml}$; cat. no: 012-15211; Wako Pure Chemical Industries, Ltd., Osaka, Japan); LeX (mouse anti-mouse P12, 1/100; cat. no: AB74035; Abcam, Cambridge, MA, USA); STn (mouse anti-mouse B72.3 TAG-72, $20 \mu \mathrm{g} / \mathrm{ml}$; cat. no: sc-20042; Santa Cruz Biotechnology, Inc., Dallas, TX, USA); Mouse IgG1 isotype control ( $2 \mathrm{ng} / \mathrm{ml}$; cat. no: MA5-14453; Thermo Fisher Scientific, Inc.), Mouse IgM isotype control (2 ng/ml; cat. no: X0942; Agilent Technologies, Inc., Santa Clara, CA, USA) The lectins used were: Mal II and SNA, (1/100; cat. nos: B-1265 and B1305; Vector Laboratories, Inc., Burlingame, CA, USA). Then, tumor cells were washed with PBS and incubated with Polyclonal Goat Anti-Mouse Phycoerythrin (PE) labeled anti-mouse immunoglobulins (5 ug/ml, cat. no: R048001; Agilent Technologies, Inc.) or Fuorescein Streptavidin (cat. no: SA-5001; Vector Laboratories, Inc.) for $30 \mathrm{~min}$ at $4^{\circ} \mathrm{C}$. Acquisition was achieved by a FACSCalibur flow cytometer (BD Biosciences, Franklin Lakes, NJ, USA) and analyzed by the FlowJo ${ }^{\circledR}$ software.

Immunohistochemistry (IHC). Staining of sections consisted of antigen retrieval with sodium citrate followed by incubation with blocking serum (Vectastain Elite ABC HRP kit (Peroxidase, Universal, cat. no: PK-6200; Vector Laboratories, Inc.). Sections were incubated with specific mAbs or PBS as negative control for $30 \mathrm{~min}$. The mAbs used were: NeuGcGM3 (mouse anti-mouse 14F7, $25 \mu \mathrm{g} / \mathrm{ml}$; produced by the Center of Molecular Immunology, La Habana, Cuba); SLeX (mouse anti-mouse SNH3, $5 \mu \mathrm{g} / \mathrm{ml}$; cat. no: 012-15211; Wako Pure Chemical Industries, Ltd.); LeX (mouse anti-mouse P12, 1/40; cat. no: AB74035; Abcam); STn (mouse anti-mouse B72.3 TAG-72, $4 \mu \mathrm{g} / \mathrm{ml}$; cat. no: sc-20042; Santa Cruz Biotechnology, Inc.). Sections were incubated with biotinylated secondary $\mathrm{Ab}$ (Vectastain Elite ABC HRP Kit (Peroxidase, Universal; cat. no: PK-6200; Vector Laboratories, Inc.) for $1 \mathrm{~h}$, followed by peroxidase-conjugated avidin-biotin complex for $1 \mathrm{~h}$. Bound antibodies were detected by incubation with diaminobenzidine substrate and tumor sections were then counterstained with haematoxylin. Results obtained by IHC were semiquantitatively evaluated, and scored by two independent observers. Specific staining intensity was compared to its negative control and graded as intense, moderate, low or negative.

Heterotopic tumor growth. At day 0, groups of at least three mice were inoculated subcutaneously in the right flank with $2 \times 10^{5}$ viable MB49 or MB49-I cells per mouse in $0.2 \mathrm{ml}$ of RPMI. Primary tumor development was monitored by palpation and tumors were measured periodically with a caliper. Tumor volume was calculated by the formula: $0.52 \mathrm{x}$ width ${ }^{2} \mathrm{x}$ length. Animals were sacrificed by cervical dislocation when subcutaneous tumor volume reached $2,000 \mathrm{~mm}^{3}$. Tumors obtained were removed, fixed with formalin, embedded in paraffin and processed for histology as previously described.

Orthotopic tumor growth. Orthotopic tumors were developed as described in Lodillinsky et al (17). Briefly, a 24 gauge polytetrafluoroethylene coated catheter was introduced into the bladder lumen through the urethra of anesthetized C57BL/6J female mice [intraperitoneal injection of ketamine $(70 \mathrm{mg} / \mathrm{kg})$ plus xylazine $(5 \mathrm{mg} / \mathrm{kg})] .2 \times 10^{4}$ cells from subconfluent MB49 or MB49-I monolayers were instilled in $100 \mu$ l RPMI. Mice were monitored twice weekly for hematuria (blood in urine during bladder palpation) and by bladder palpation. Two weeks after tumor cell inoculation the animals were sacrificed by cervical dislocation (17). Tumors obtained were removed, fixed with formalin, embedded in paraffin and processed for histology as previously described.

Statistical analysis. Results described in this work are expressed as median with interquartile range. Statistical analyses were carried out using GraphPad Prism v.6.0 statistical software (GraphPad, Inc., La Jolla, CA, USA). Normal distribution of data was evaluated using the D'Agostino and Pearson omnibus normality test, for comparisons between experimental groups, we used Mann Whitney test. $\mathrm{P}<0.05$ was considered to indicate a statistically significant difference.

\section{Results}

Glycans expression in cell line monolayers. The first approach in the characterization of selected glycans expression of the murine bladder cancer cell lines was the analysis of the binding of two lectins that recognize sialic acids bound to glycoconjugates. Mal II is a lectin derived from Maackia amurensis and has affinity for $\alpha 2,3$-linked sialic acid. This type of linkage is associated with the presence of SLeX (Fig. 1). SNA lectin derives from Sambucus nigra and binds to $\alpha 2,6$-linked sialic acid, present in STn (Fig. 1). When the binding of Mal II was evaluated by FACS, MB49 showed a higher fluorescence intensity than MB49-I, indicating that it possesses more sialic acids with $\alpha 2,3$ linkage (Fig. 2A). Same results were obtained when SNA was evaluated, showing more binding to MB49 cells in relation to MB49-I (Fig. 2B).

The expression of the glycans LeX, SLeX, STn and NGcGM3 (Fig. 1) in the murine bladder cancer cell lines MB49 and MB49-I was then evaluated by FACS. Representative dot plots of the percentage of positive cells of these four glycans for MB49 and MB49-I cells respectively, are shown in Fig. 3A and B. The percentage of antigen expression was considered 'high' when more than $50 \%$ of positive events were observed, 'moderate' when it was measured between 30 to $50 \%$ and 'low' when it was less than $30 \%$ but more than $10 \%$. The glycan LeX was not detected in any of the cell lines. On the other hand, both cell lines were positive for SLeX, with a high expression displayed in MB49 and a moderate one in MB49-I. Median fluorescence intensity values (MFI) were 47.42 and 8.99 for MB49 and MB49-I respectively. MB49 also exhibited a high expression of STn, showing a MFI of 14.36, while MB49-I was considered negative for this antigen (MFI: 0.2). The results of the evaluation of SLex and STn by FACS were in accordance with the results obtained in the lectins binding assay. In relation to NGcGM3, MB49 showed low expression of this ganglioside, while MB49-I was moderately positive.

Glycan expression in three-dimensional spheroids. Three-dimensional spheroids were analyzed by IHC. Intensity of color developed by the staining with each $\mathrm{mAb}$ was compared to its negative control assigning one 


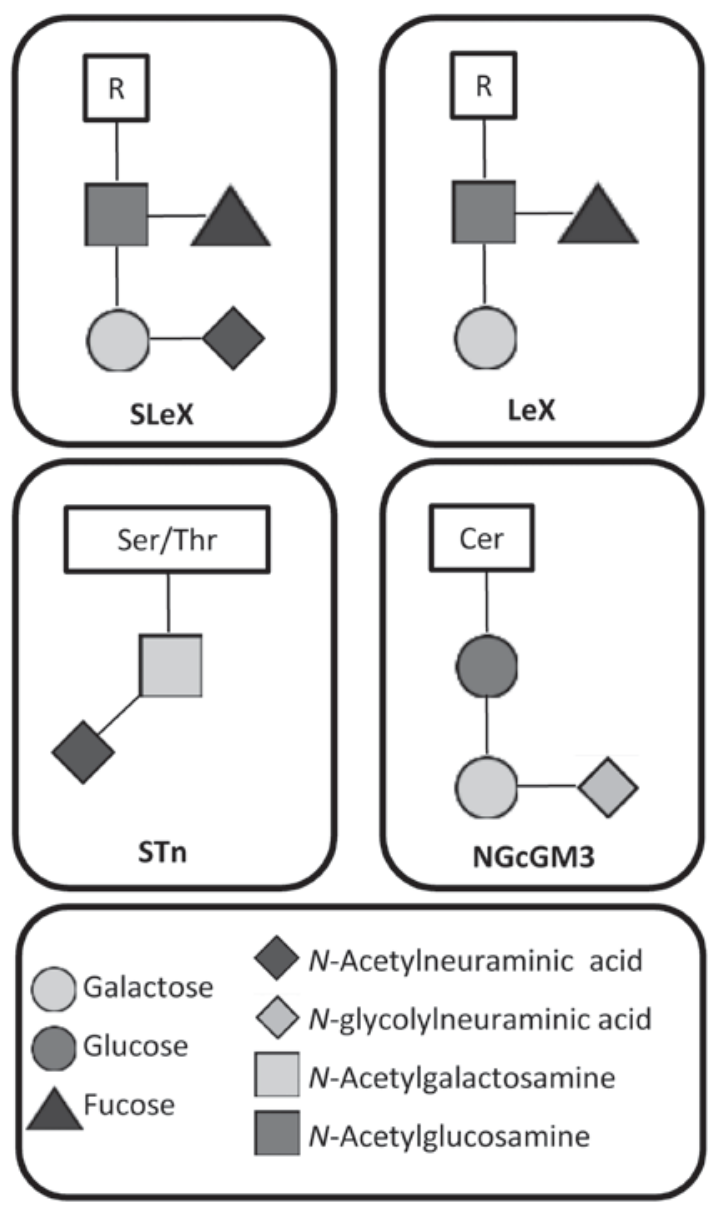

Figure 1. Monosaccharide structure of the glycans analyzed in the present study. LeX, Lewis X; SLeX, Sialyl Lewis X; STn, Sialyl Tn; NGcGM3, $\mathrm{N}$-glycolyl GM3. The letter R represents any structure of a protein or lipid.

of the four scores described in the Materials and Methods section. In the case of tumors spheroids, the expression of the glycan LeX was considered negative in both cell lines, while SLeX showed an intense staining in MB49 spheroids and a moderate one in MB49-I. The antigen STn had a moderate expression but only in MB49 spheroids. On the other hand, the ganglioside NGcGM3 was highly expressed in spheroids derived from both cell lines. Representative photos are presented in Fig. 4A and B.

Glycan expression in tumors. Regarding heterotopic tumors, NGcGM3 was the only glycan that was intensely expressed in both MB49 and MB49-I. None of the three other glycans were detected in this type of tumors (Fig. 5A and B). On the other hand, the analysis of glycans expression in orthotopic tumors revealed an intense staining of SLeX in MB49 tumors and a moderate one in MB49-I. Both MB49 and MB49-I tumors highly expressed NGcGM3; but STn and LeX were not detected whatsoever (Fig. 5C and D).

NGcGM3 expression in T24 human bladder cancer cells. Taking into account the obtained results regarding the expression of NGcGM3 in murine cell lines and the fact that there are no reports of this glycan in human bladder cancer, we decided to evaluate the expression of this ganglioside in the cell line T24. NGcGM3 expression was analyzed either in cell
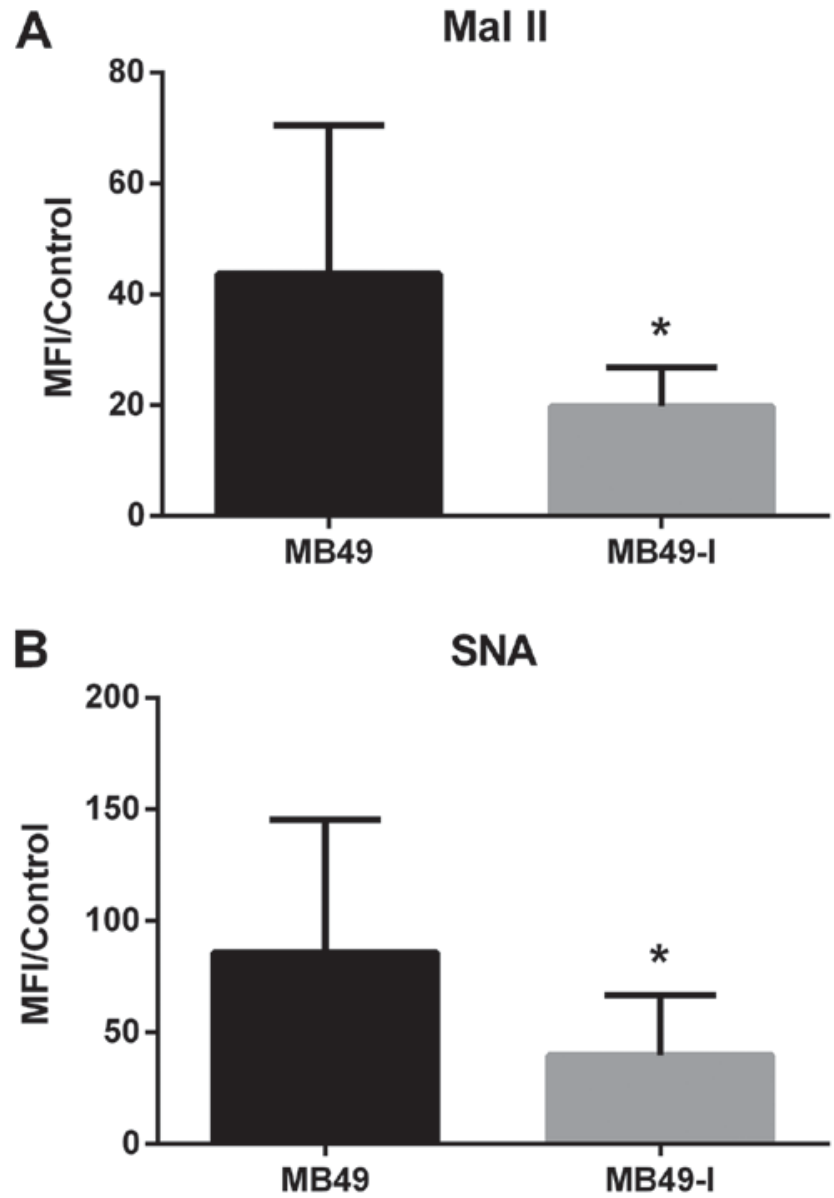

Figure 2. Evaluation of lectins (A) Mal II and (B) SNA binding to MB49 and MB49-I cell lines by FACS. Results correspond to median fluorescence intensity relative to negative control (PBS) of five independent experiments. Data are expressed as median with interquartile range; Mann Whitney test ${ }^{*} \mathrm{P}<0.05$. FACS, fluorescence activated cell sorting; MFI, Median fluorescence intensity values.

monolayer or in three-dimensional tumor spheroids by FACS or IHC respectively. NGcGM3 was moderately expressed in this cell line (Fig. 6A) with a MFI of 6.47. Accordingly, spheroids derived from T24 cell line revealed an intense staining of this glycan (Fig. 6B).

\section{Discussion}

Glycans are targets of many approved and developing anticancer treatments and thus it is important to have preclinical models that express these antigens recreating human disease in order to evaluate and validate anticancer therapies. Tumor cell lines have been widely used for in vitro studies, but this approach does not resemble the 3D properties of tumors in physiological conditions, including interactions with immune cells and tumor microenvironment $(3,47)$. A first step to shorten the distance between in vitro and in vivo studies is the three-dimensional multicellular tumor spheroid model, where cells adhere to each other forming a spherical cell mass with different cell populations and no artificial substrate for cell attachment. Tumor spheroids generally have a population of proliferating cells in the outer region and a group of quiescent and necrotic cells in the central zone; a phenomenon that occurs due to the existent 
A

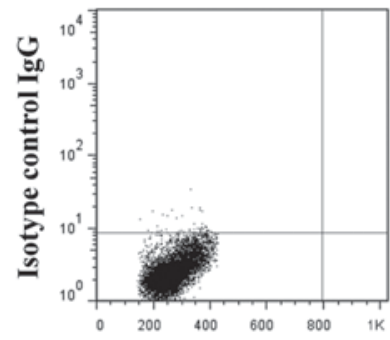

MB49
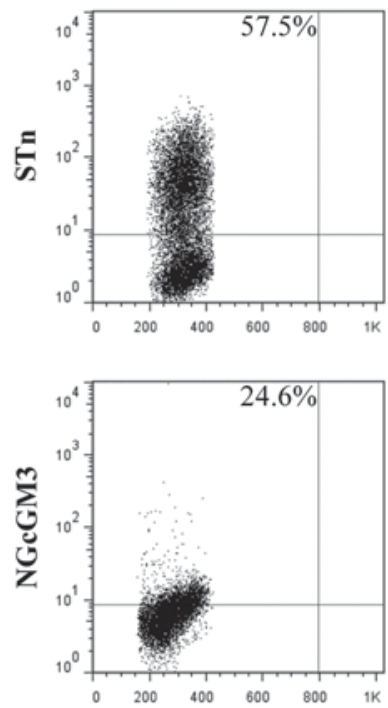
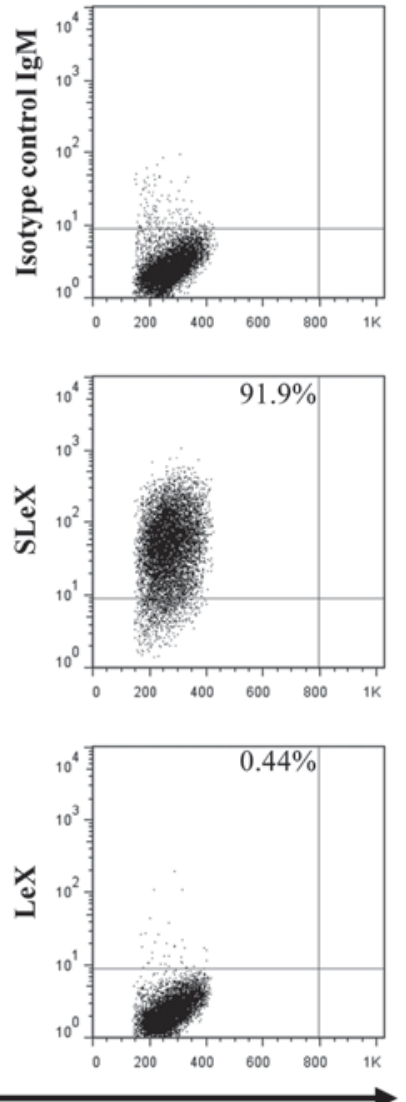

FSC-H
B

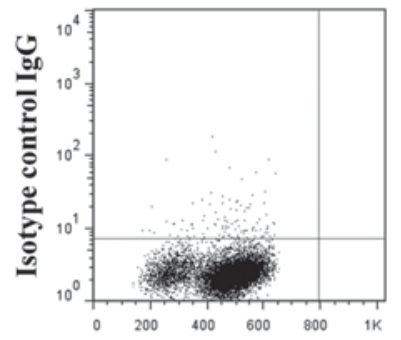

MB49-I
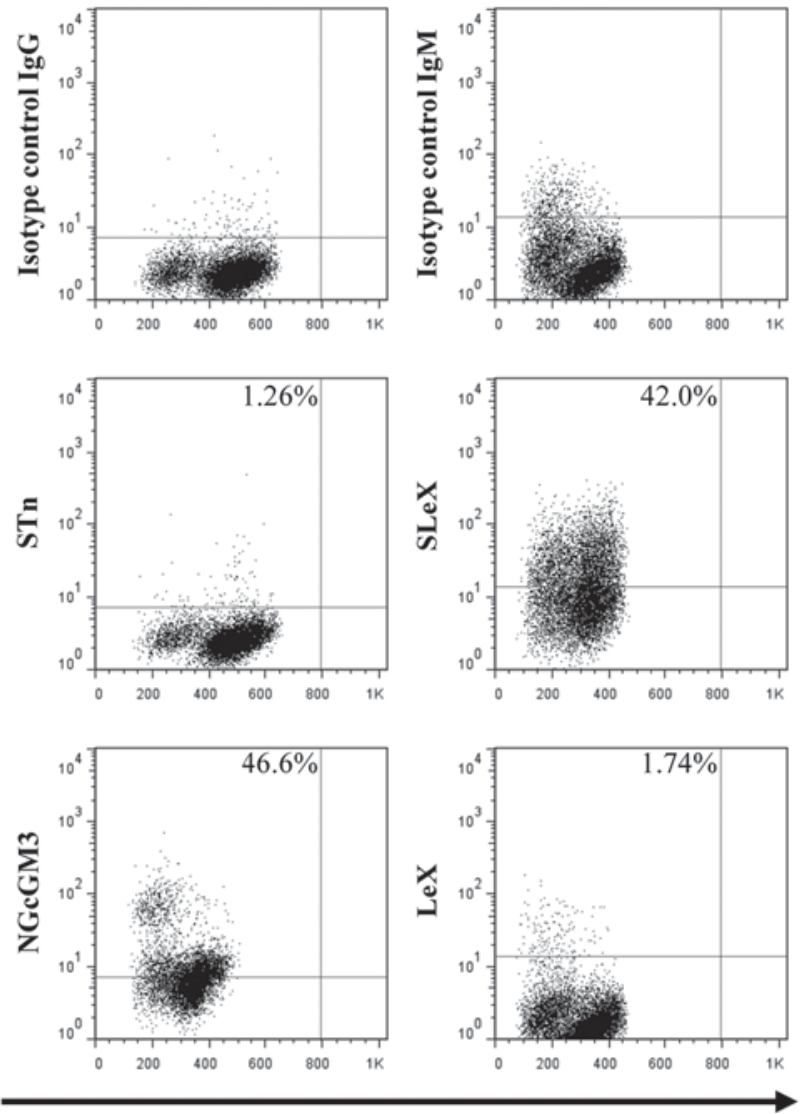

FSC-H

Figure 3. Evaluation of glycan expression in (A) MB49 and (B) MB49-I cell lines by FACS. The percentage of positive cells for each glycan is indicated in the graphs. Representative dot plots of at least three independent experiments. FSC-H, forward side scatter.
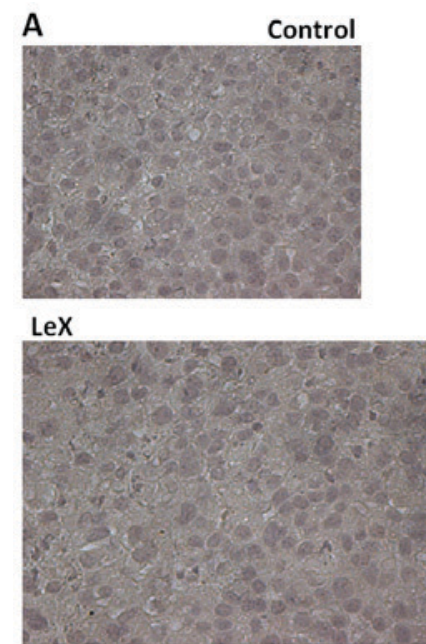

STn

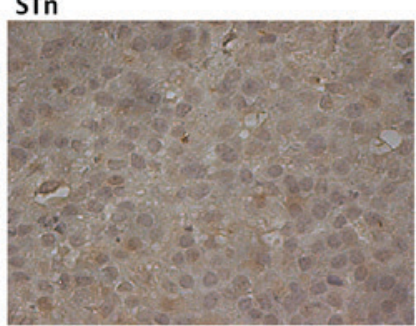

SLeX

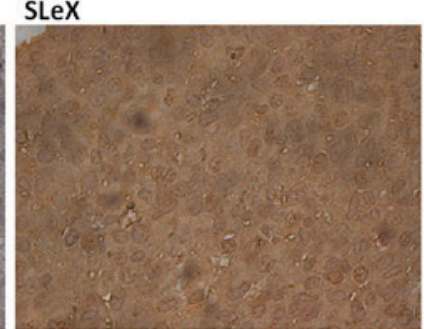

NGcGM3

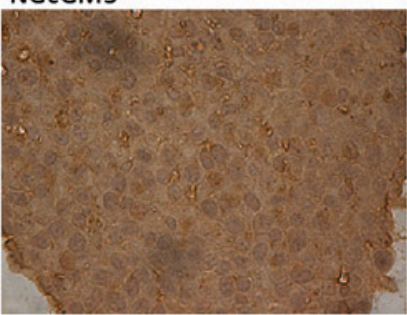

B

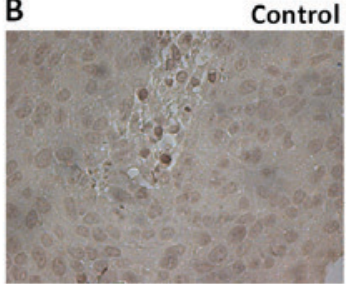

LeX

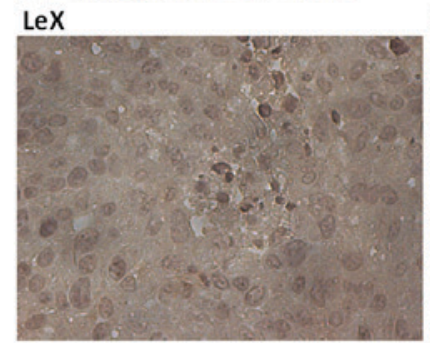

SLeX

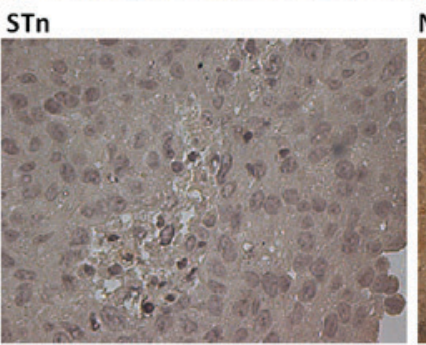

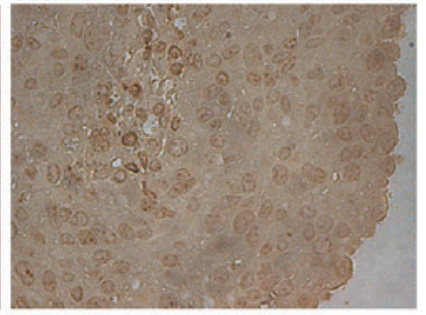

NGcGM3

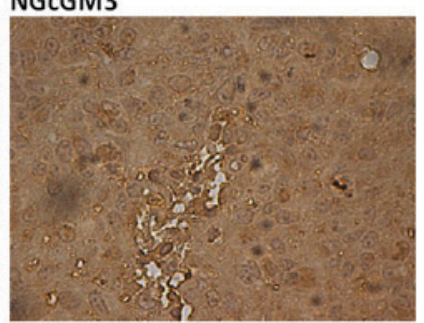

Figure 4. Evaluation of glycan expression in (A) MB49 and (B) MB49-I three-dimensional tumor spheroids by IHC. Photos are representative of at least three experiments. Original magnification, $\mathrm{x} 400$. 
A
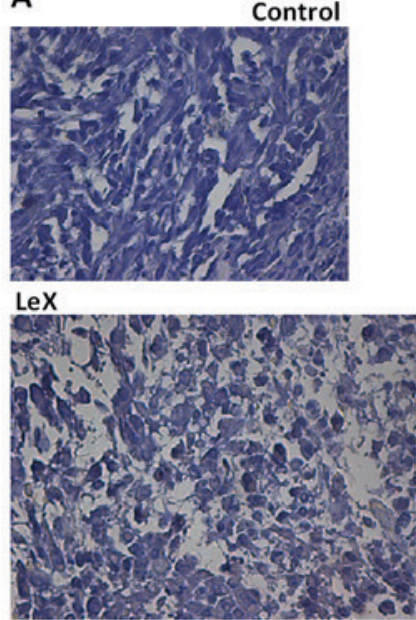

STn

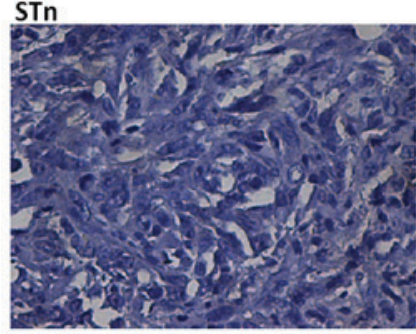

C

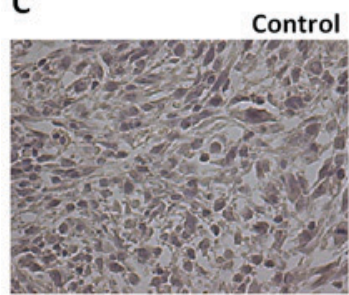

LeX

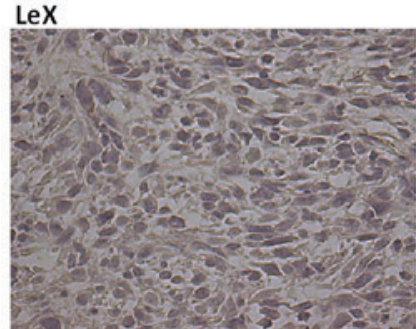

STn

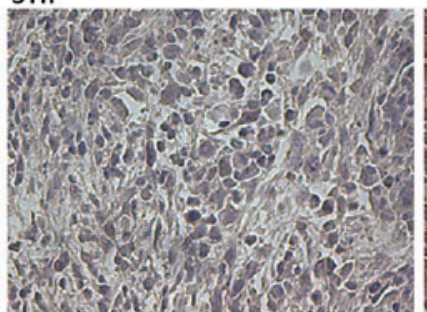

SLeX

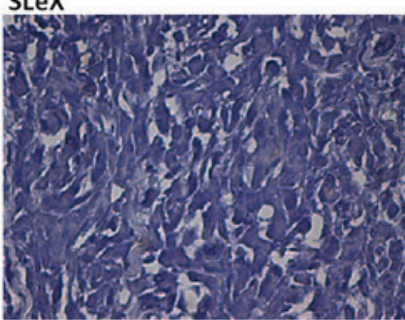

NGcGM3

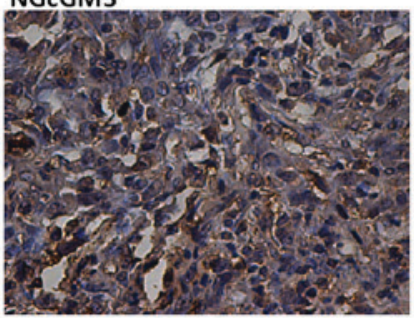

SLeX

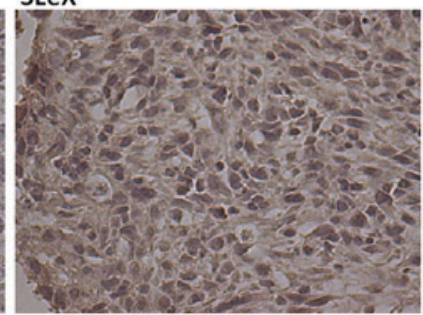

NGcGM3

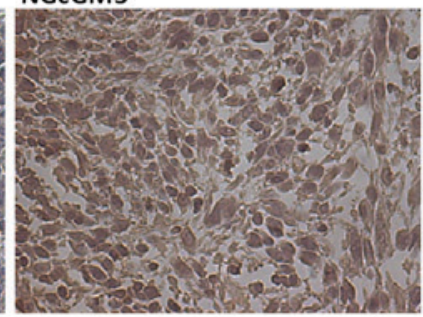

B

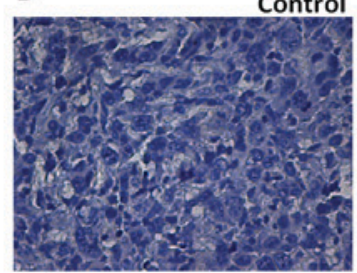

LeX

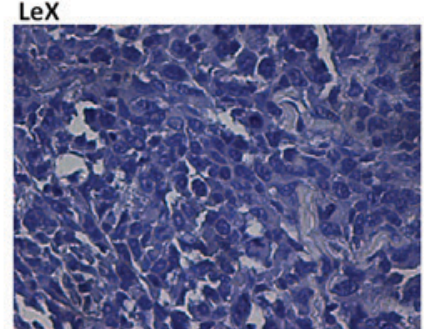

STn

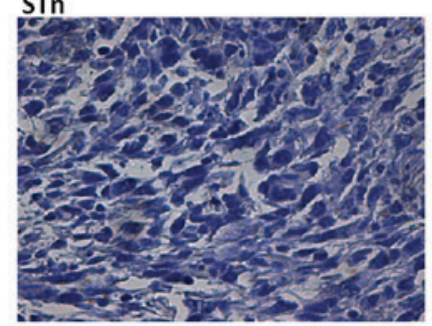

SLeX

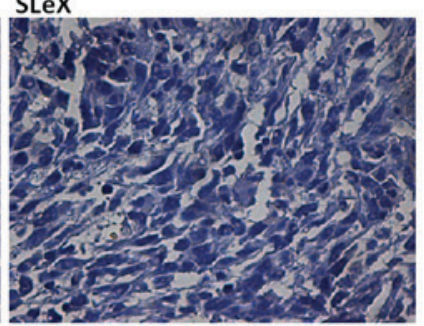

NGcGM3

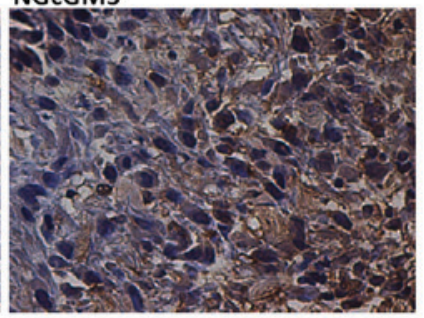

D

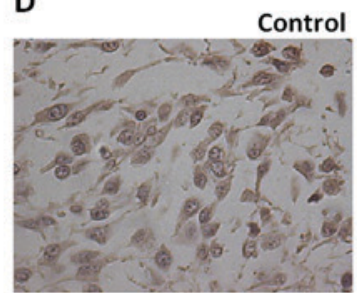

LeX

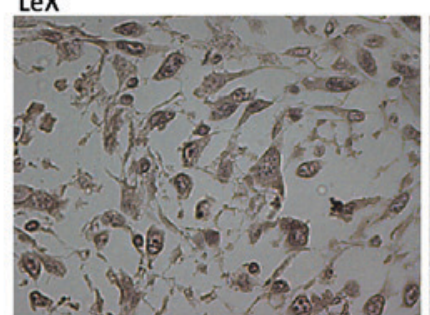

SLeX

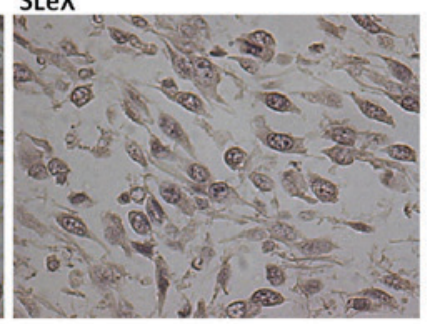

STn

NGcGM3

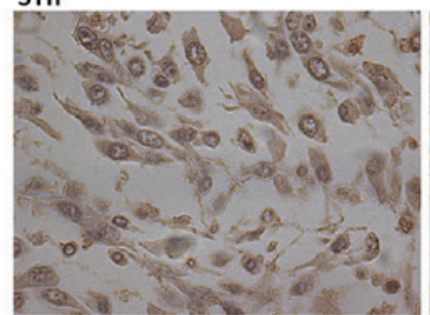

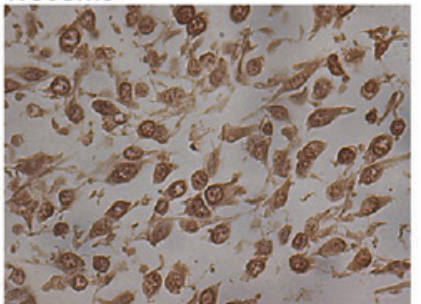

Figure 5. Evaluation of glycan expression in tumors by IHC. (A) MB49 heterotopic tumors; (B) MB49-I heterotopic tumors; (C) MB49 orthotopic tumors; (D) MB49-I orthotopic tumors. Photos are representative of at least three experiments. Original magnification, $\mathrm{x} 400$.

gradient of nutrients and oxygen $(3,48,49)$. Thus, the growth of tumor spheroids can better recreate tumor cellular heterogeneity, cell-cell interactions and spatial structure, with the advantage of being a low cost and short duration assay $(3,47,49)$.

Regarding in vivo studies, the development of heterotopic tumors in mice, mainly subcutaneous tumors, has been extensively used in cancer research due to its many advantages, such as easy manipulation and the ability to monitor tumor growth. However, it is well reported that this surrogate microenvironment affects the tumor in a different manner compared to the tumor growing in its orthotopic site (50-52). Hence, the importance of characterizing each model. 

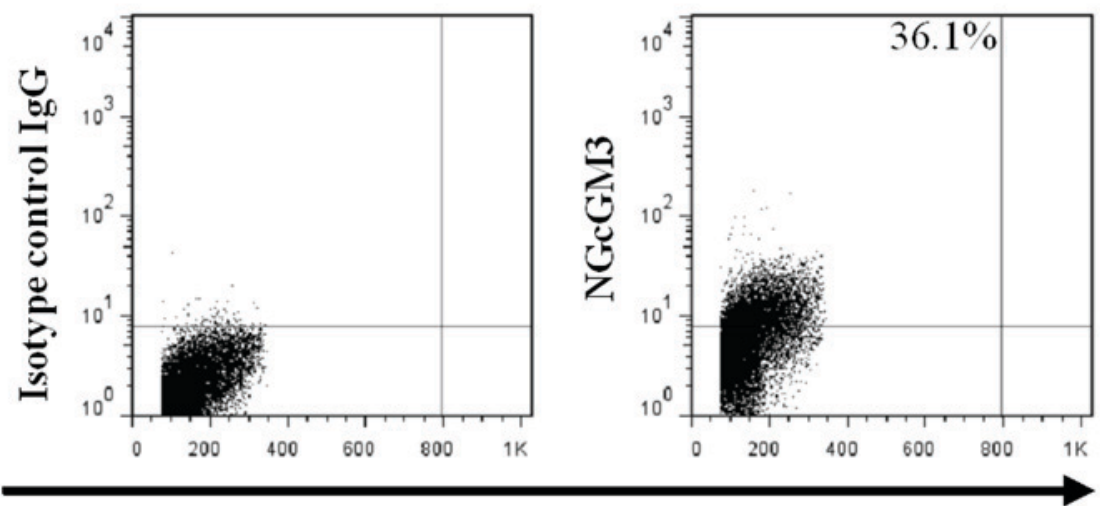

FSC-H

\section{B Control}

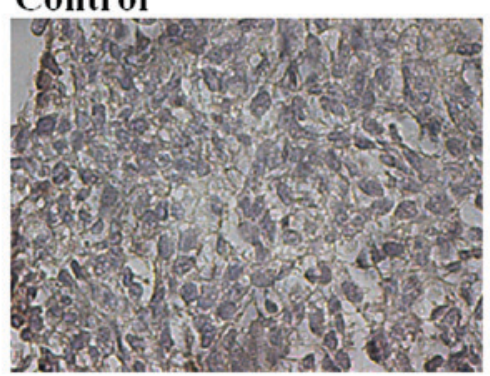

\section{NGcGM3}

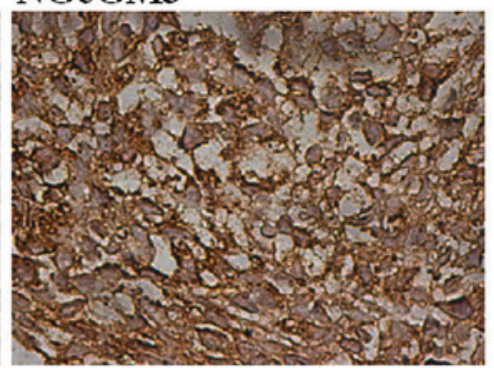

Figure 6. Evaluation of NGcGM3 expression in (A) T24 cell line by FACS; Representative dot plots of at least three independent experiments. (B) T24 three-dimensional tumor spheroids by IHC. Photos are representative of at least three experiments. Original magnification, $\mathrm{x} 400$. The percentage of positive cells for each glycan is indicated in the graphs. FSC-H, forward side scatter.

The use of murine cancer cell lines and the growth of tumors in immunocompetent mice allow the study of the effect of the microenvironment and the immune system on tumor progression, among other advantages. In the case of bladder cancer, MB49 and MB49-I are the only murine tumor cell lines available, thus it is essential to better characterize them in order to describe and establish their in vitro and in vivo behavior. Taking into account the importance of glycans in tumor biology, and considering they can act as targets of therapies and biomarkers, we evaluated the expression of LeX, SLeX, STn and NGcGM3 in MB49 and MB49-I, including monolayer cultures, three-dimensional multicellular spheroids, heterotopic and orthotopic tumors developed from these cell lines.

The glycan LeX has been proposed as a biomarker of urothelial carcinoma (30-32). Nevertheless, the evaluation of LeX expression in both cell lines revealed no detectable presence of this antigen in either monolayers or spheroids nor in tumors; rendering this model useless for the study of the role of this glycan in bladder cancer biology (Table I). Another relevant glycan evaluated was SLeX, which has been associated with invasiveness and metastatic capacity in various types of cancer, including bladder cancer $(20,25,34,35,53)$. Its presence was detected in both cell lines, and also in spheroids and orthotopic tumors. No expression was found in heterotopic tumors whatsoever. MB49 and MB49-I appear as useful models for the study of SLeX in bladder cancer, both in vitro, including the spheroids model, and in vivo, with the development of orthotopic tumors (Table I). The neo-antigen STn was also associated with aggressiveness and poor prognosis in human bladder cancer (36-38). The presence of this glycan was only confirmed in MB49 monolayers and spheroids. Both heterotopic and orthotopic tumors derived from this cell line lost the expression of this antigen (Table IA), confirming the importance of the role of the microenvironment in the glycophenotype. In relation to MB49-I, it appears that during the development of this cell line the expression of STn was not essential in tumor malignancy and was lost (Table IB). Even though this antigen is present in more than $80 \%$ of human carcinomas, including urothelial carcinomas (54), there are no reports regarding its expression in tumor cell lines. Furthermore, the majority of the models used to study this antigen include the induced overexpression of the enzymes involved in its synthesis $(38,39,55-57)$. Therefore, MB49 cell line maintained in vitro either as a monolayer or as spheroids, appear to be valuable models for the in vitro study of STn. As mentioned before, three-dimensional multicellular spheroids can better recreate tumor cellular growth condition in vitro. Considering our results regarding STn expression, tumor spheroids in particular seem to be a reliable tumor growth model for analyzing the relevance of this glycan in bladder cancer.

$\mathrm{N}$-glycolylneuraminic acid (NeuGc) is not found in human tissues due to a deletion in the CMP-N-acetylneuraminic acid hydroxylase (CMAH) gene. CMAH is the enzyme responsible for the synthesis of this sialic acid $(58,59)$ which is then incorporated to different membrane glycoconjugates, such as the ganglioside NGcGM3, by the action of 
Table I. Glycan expression in MB49 and MB49-I cell lines in different growth conditions.

A, MB49

\begin{tabular}{lllll}
\hline Antigen & Monolayers & Tumor spheroids & Heterotopic tumors & Orthotopic tumors \\
\hline LeX & Negative & Negative & Negative & Negative \\
SLeX & High & Intense & Negative & Intense \\
STn & High & Moderate & Negative & Negative \\
NGcGM3 & Low & Intense & Intense & Intense \\
\hline
\end{tabular}

B, MB49-I

\begin{tabular}{|c|c|c|c|c|}
\hline Antigen & Monolayers & Tumor spheroids & Heterotopic tumors & Orthotopic tumors \\
\hline LeX & Negative & Negative & Negative & Negative \\
\hline SLeX & Moderate & Moderate & Negative & Moderate \\
\hline STn & Negative & Negative & Negative & Negative \\
\hline NGcGM3 & Medium & Intense & Intense & Intense \\
\hline
\end{tabular}

LeX, Lewis X; SLeX, Sialyl Lewis X; STn, Sialyl Tn; NGcGM3, N-glycolyl GM3.

sialyltransferases. Even though human tissues do not express NGcGM3, it is considered a tumor neo-antigen in many types of cancer (40-45). It is proposed that tumor cells can incorporate NeuGc from dietary sources (60) due to the overexpression of the sialic acid transporter sialin under hypoxic conditions (61). There are no reports concerning the expression of NGcGM3 in human or murine bladder cancer. In our hands, MB49 and MB49-I expressed this ganglioside in all the growth conditions evaluated (Table I). Moreover, the evaluation of the presence of this antigen in the human bladder cancer cell line T24 and its spheroids turned out to be positive. The assessment of the expression of NGcGM3 in human bladder cancer cell lines and patient's tumors is of great importance, considering the relevance of this ganglioside in tumor biology, its differential expression in tumor cells over normal tissues and the existence of NGcGM3-directed therapies $(62,63)$.

The characterization of murine cell lines is of great importance so as to find models that resemble human diseases. MB49 and MB49-I are currently the only two murine cell lines available for the development of preclinical bladder cancer models. In this work, we demonstrate that glycan expression may substantially change depending on growth conditions. The three-dimensional tumor spheroid model showed a pattern of glycan expression similar to the observed in cell line monolayers. On the other hand, heterotopic tumors, although useful for their simplicity in comparison with orthotopic ones, do not satisfactorily recreate the original microenvironment, thus affecting the tumor expression of certain glycans. Among in vivo models, the setting that better resembled tumor glycan expression for these cell lines were the orthotopic tumors, and we consider that it should be the first choice for in vivo studies when possible. This is the first work that reports the expression of relevant bladder cancer-associated glycans in the two murine cell lines available for the development of preclinical studies in bladder cancer. Although studies of the biological role of these glycans in MB49 and MB49-I are necessary to confirm their similarity to human bladder cancer, this work also provides useful information when choosing a model for the evaluation and validation of anticancer therapies based on these glycans in this type of cancer.

\section{Acknowledgements}

Not applicable.

\section{Funding}

The present study was partially funded by National University of Quilmes (grant no. PUNQ 2015-2019. Res 1398/15).

\section{Availability of data and materials}

The datasets used and/or analyzed during the present study are available from the corresponding author on reasonable request.

\section{Authors' contributions}

MA, MRG, AME and VIS, contributed to the study conception and design of the experiments. MA, HAC and CAG performed experiments. HAC acquired data. MP contributed with the statistical analysis of the data. DB and AME contributed to the development of the orthotopic tumors in mice. MA and VIS participated in the writing of the manuscript. MA, MP, MRG and VIS drafted, edited, critically revised and approved final version of manuscript.

\section{Ethics approval and consent to participate}

All protocols were approved by the Institutional Committee for the Care and Use of Laboratory Animals of Quilmes National University. 


\section{Patient consent for publication}

Not applicable.

\section{Competing interests}

All authors declare that they have no competing interests.

\section{Authors' information}

HAC is a research fellow of ANPCyT (Argentina). MA, MP and DB are research fellows and AME, MRG and VIS are members of the National Research Council (CONICET, Argentina). ORCID ID MA: 0000-0003-1533-3062. ORCID ID MRG: 0000-0001-8395-9222. ORCID ID VIS: 0000-0002-3913-3658.

\section{References}

1. Bray F, Ren JS, Masuyer E and Ferlay J: Global estimates of cancer prevalence for 27 sites in the adult population in 2008. Int J Cancer 132: 1133-1145, 2013

2. Kirkali Z, Chan T, Manoharan M, Algaba F, Busch C, Cheng L, Kiemeney L, Kriegmair M, Montironi R, Murphy WM, et al: Bladder cancer: Epidemiology, staging and grading, and diagnosis. Urology 66 (6 Suppl 1): S4-S34, 2005

3. Huang BW and Gao JQ: Application of 3D cultured multicellular spheroid tumor models in tumor-targeted drug delivery system research. J Control Release 270: 246-259, 2018.

4. Tabayoyong $\mathrm{W}$ and Gao J: The emerging role of immunotherapy in advanced urothelial cancers. Curr Opin Oncol 30: 172-180, 2018.

5. Nadal R and Apolo AB: Overview of current and future adjuvant therapy for muscle-invasive urothelial carcinoma. Curr Treat Options Oncol 19: 36, 2018.

6. Redelman-Sidi G, Glickman MS and Bochner BH: The mechanism of action of BCG therapy for bladder cancer-a current perspective. Nat Rev Urol 11: 153-162, 2014.

7. Bladder Cancer Treatment $\left(\mathrm{PDQ}^{\circledR}\right)$ : Health Professional Version: PDQ cancer information summaries. Bethesda (MD), 2002.

8. Rouanne M, Loriot Y, Lebret T and Soria JC: Novel therapeutic targets in advanced urothelial carcinoma. Crit Rev Oncol Hematol 98: 106-115, 2016.

9. Rouanne M, Roumiguié M, Houédé N, Masson-Lecomte A, Colin P, Pignot G, Larré S, Xylinas E, Rouprêt M and Neuzillet Y: Development of immunotherapy in bladder cancer: Present and future on targeting $\mathrm{PD}(\mathrm{L}) 1$ and CTLA-4 pathways. World J Urol 36: 1727-1740, 2018.

10. Kobayashi T, Owczarek TB, McKiernan JM and Abate-Shen C: Modelling bladder cancer in mice: Opportunities and challenges. Nat Rev Cancer 15: 42-54, 2015.

11. Smolensky D, Rathore K and Cekanova M: Molecular targets in urothelial cancer: Detection, treatment, and animal models of bladder cancer. Drug Des Devel Ther 10: 3305-3322, 2016.

12. Zhang N, Li D, Shao J and Wang X: Animal models for bladder cancer: The model establishment and evaluation (Review). Oncol Lett 9: 1515-1519, 2015.

13. Summerhayes IC and Franks LM: Effects of donor age on neoplastic transformation of adult mouse bladder epithelium in vitro. J Natl Cancer Inst 62: 1017-1023, 1979.

14. Chan E, Patel A, Heston W and Larchian W: Mouse orthotopic models for bladder cancer research. BJU Int 104: 1286-1291, 2009.

15. De Boer EC, Teppema JS, Steerenberg PA and De Jong WH: Retrovirus type $\mathrm{C}$ in the mouse bladder carcinoma cell line MBT-2. J Urol 163: 1999-2001, 2000.

16. Kasman L and Voelkel-Johnson C: An orthotopic bladder cancer model for gene delivery studies. J Vis Exp: 50181, 2013.

17. Lodillinsky C, Rodriguez V, Vauthay L, Sandes E, Casabé A and Eiján AM: Novel invasive orthotopic bladder cancer model with high cathepsin $\mathrm{B}$ activity resembling human bladder cancer. J Urol 182: 749-755, 2009.

18. Chen F, Zhang G, Cao Y, Hessner MJ and See WA: MB49 murine urothelial carcinoma: Molecular and phenotypic comparison to human cell lines as a model of the direct tumor response to bacillus Calmette-Guerin. J Urol 182: 2932-2937, 2009.
19. Fabris VT, Lodillinsky C, Pampena MB, Belgorosky D, Lanari C and Eiján AM: Cytogenetic characterization of the murine bladder cancer model MB49 and the derived invasive line MB49-I. Cancer Genet 205: 168-176, 2012.

20. Varki A, Kannagi R, Toole B and Stanley P: Glycosylation Changes in Cancer. In: rd, Varki A, Cummings RD, Esko JD, Stanley P, Hart GW, et al: editors. Essentials of Glycobiology. Cold Spring Harbor (NY): pp597-609, 2015.

21. Vajaria BN and Patel PS: Glycosylation: A hallmark of cancer? Glycoconj J 34: 147-156, 2017.

22. Pinho SS and Reis CA: Glycosylation in cancer: Mechanisms and clinical implications. Nat Rev Cancer 15: 540-555, 2015.

23. Pearce OMT: Cancer glycan epitopes: Biosynthesis, structure and function. Glycobiology 28: 670-696, 2018.

24. Tuccillo FM, de Laurentiis A, Palmieri C, Fiume G, Bonelli P, Borrelli A, Tassone P, Scala I, Buonaguro FM, Quinto I and Scala G: Aberrant glycosylation as biomarker for cancer: Focus on CD43. Biomed Res Int 2014: 742831, 2014.

25. Heimburg-Molinaro J, Lum M, Vijay G, Jain M, Almogren A and Rittenhouse-Olson $\mathrm{K}$ : Cancer vaccines and carbohydrate epitopes. Vaccine 29: 8802-8826, 2011.

26. Rabu C, McIntosh R, Jurasova Z and Durrant L: Glycans as targets for therapeutic antitumor antibodies. Future Oncol 8: 943-960, 2012.

27. Segatori VI, Otero LL, Fernandez LE, Gomez DE, Alonso DF and Gabri MR: Antitumor protection by NGcGM3/VSSP vaccine against transfected B16 mouse melanoma cells overexpressing N-glycolylated gangliosides. In Vivo 26: 609-617, 2012.

28. Segatori VI, Vazquez AM, Gomez DE, Gabri MR and Alonso DF: Preclinical evaluation of racotumomab, an anti-idiotype monoclonal antibody to N-glycolyl-containing gangliosides, with or without chemotherapy in a mouse model of non-small cell lung cancer. Front Oncol 2: 160, 2012.

29. Steentoft C, Migliorini D, King TR, Mandel U, June CH and Posey AD Jr: Glycan-directed CAR-T cells. Glycobiology 28: 656-669, 2018

30. Ohyama C: Glycosylation in bladder cancer. Int J Clin Oncol 13: 308-313, 2008

31. Parker J and Spiess PE: Current and emerging bladder cancer urinary biomarkers. ScientificWorldJournal 11: 1103-1112, 2011.

32. Dal Moro F, Valotto C, Guttilla A and Zattoni F: Urinary markers in the everyday diagnosis of bladder cancer. Urologia 80: 265-275, 2013.

33. Burchardt M, Burchardt T, Shabsigh A, De La Taille A, Benson MC and Sawczuk I: Current concepts in biomarker technology for bladder cancers. Clin Chem 46: 595-605, 2000.

34. Fujii Y, Yoshida M, Chien LJ, Kihara K, Kageyama Y, Yasukochi Y and Oshima H: Significance of carbohydrate antigen sialyl-Lewis $\mathrm{X}$, sialyl-Lewis $\mathrm{A}$, and possible unknown ligands to adhesion of human urothelial cancer cells to activated endothelium. Urol Int 64: 129-133, 2000.

35. Numahata K, Satoh M, Handa K, Saito S, Ohyama C, Ito A, Takahashi T, Hoshi S, Orikasa S and Hakomori SI: Sialosyl-Le(x) expression defines invasive and metastatic properties of bladder carcinoma. Cancer 94: 673-685, 2002

36. Lima L, Neves M, Oliveira MI, Dieguez L, Freitas R, Azevedo R, Gaiteiro C, Soares J, Ferreira D, Peixoto A, et al: Sialyl-Tn identifies muscle-invasive bladder cancer basal and luminal subtypes facing decreased survival, being expressed by circulating tumor cells and metastases. Urol Oncol 35: 675.e1-675.e8, 2017.

37. Cotton S, Azevedo R, Gaiteiro C, Ferreira D, Lima L, Peixoto A, Fernandes E, Neves M, Neves D, Amaro T, et al: Targeted O-glycoproteomics explored increased sialylation and identified MUC16 as a poor prognosis biomarker in advanced-stage bladder tumours. Mol Oncol 11: 895-912, 2017.

38. Peixoto A, Fernandes E, Gaiteiro C, Lima L, Azevedo R, Soares J, Cotton S, Parreira B, Neves M, Amaro T, et al: Hypoxia enhances the malignant nature of bladder cancer cells and concomitantly antagonizes protein O-glycosylation extension. Oncotarget 7: 63138-63157, 2016.

39. Severino PF, Silva M, Carrascal M, Malagolini N, Chiricolo M, Venturi G, Astolfi A, Catera M, Videira PA and Dall'Olio F: Expression of sialyl-Tn sugar antigen in bladder cancer cells affects response to Bacillus Calmette Guérin (BCG) and to oxidative damage. Oncotarget 8: 54506-54517, 2017.

40. Scursoni AM, Galluzzo L, Camarero S, Lopez J, Lubieniecki F, Sampor C, Segatori VI, Gabri MR, Alonso DF, Chantada G and de Dávila MT: Detection of N-glycolyl GM3 ganglioside in neuroectodermal tumors by immunohistochemistry: An attractive vaccine target for aggressive pediatric cancer. Clin Dev Immunol 2011: 245181, 2011.

41. Malykh YN, Schauer R and Shaw L: N-Glycolylneuraminic acid in human tumours. Biochimie 83: 623-634, 2001. 
42. Carr A, Mullet A, Mazorra Z, Vázquez AM, Alfonso M, Mesa C Rengifo E, Pérez R and Fernández LE: A mouse IgG1 monoclonal antibody specific for $\mathrm{N}$-glycolyl GM3 ganglioside recognized breast and melanoma tumors. Hybridoma 19: 241-247, 2000.

43. van Cruijsen H, Ruiz MG, van der Valk P, de Gruijl TD and Giaccone G: Tissue micro array analysis of ganglioside $\mathrm{N}$-glycolyl GM3 expression and signal transducer and activator of transcription (STAT)-3 activation in relation to dendritic cell infiltration and microvessel density in non-small cell lung cancer. BMC Cancer 9: 180, 2009.

44. Blanco R, Domínguez E, Morales O, Blanco D, Martínez D, Rengifo CE, Viada C, Cedeño M, Rengifo E and Carr A: Prognostic significance of N-Glycolyl GM3 ganglioside expression in non-small cell lung carcinoma patients: New evidences. Patholog Res Int 2015: 132326, 2015.

45. Vázquez AM, Hernández AM, Macías A, Montero E, Gómez DE, Alonso DF, Gabri MR and Gómez RE: Racotumomab: An anti-idiotype vaccine related to $\mathrm{N}$-glycolyl-containing gangliosides-preclinical and clinical data. Front Oncol 2: 150, 2012.

46. Yuhas JM, Li AP, Martinez AO and Ladman AJ: A simplified method for production and growth of multicellular tumor spheroids. Cancer Res 37: 3639-3643, 1977.

47. Ferreira LP, Gaspar VM and Mano JF: Design of spherically structured 3D in vitro tumor models-Advances and prospects. Acta Biomaterialia 75: 11-34, 2018.

48. Kucinska M, Murias M and Nowak-Sliwinska P: Beyond mouse cancer models: Three-dimensional human-relevant in vitro and non-mammalian in vivo models for photodynamic therapy. Mutat Res 773: 242-262, 2017.

49. Lu H and Stenzel MH: Multicellular tumor spheroids (MCTS) as a 3D in vitro evaluation tool of nanoparticles. Small 14: e1702858, 2018.

50. Du Q, Jiang L, Wang XQ, Pan W, She FF and Chen YL: Establishment of and comparison between orthotopic xenograft and subcutaneous xenograft models of gallbladder carcinoma. Asian Pac J Cancer Prev 15: 3747-3752, 2014.

51. Zhang Y, Zhang GL, Sun X, Cao KX, Ma C, Nan N, Yang GW, Yu MW and Wang XM: Establishment of a murine breast tumor model by subcutaneous or orthotopic implantation. Oncol Lett 15: 6233-6240, 2018.

52. Schrauwen S, Coenegrachts L, Depreeuw J, Luyten C, Verbist G, Debruyne D, Vergote I, Lambrechts D and Amant F: Microsatellite instable and microsatellite stable primary endometrial carcinoma cells and their subcutaneous and orthotopic xenografts recapitulate the characteristics of the corresponding primary tumor. Int J Gynecol Cancer 25: 363-371, 2015.

53. Kajiwara H, Yasuda M, Kumaki N, Shibayama T and Osamura Y: Expression of carbohydrate antigens (SSEA-1, sialyl-Lewis X, DU-PAN-2 and CA19-9) and E-selectin in urothelial carcinoma of the renal pelvis, ureter, and urinary bladder. Tokai J Exp Clin Med 30: 177-182, 2005.

54. Munkley J: The role of Sialyl-Tn in cancer. Int J Mol Sci 17: 275, 2016.

55. Ozaki H, Matsuzaki H, Ando H, Kaji H, Nakanishi H, Ikehara $Y$ and Narimatsu H: Enhancement of metastatic ability by ectopic expression of ST6GalNAcI on a gastric cancer cell line in a mouse model. Clin Exp Metastasis 29: 229-238, 2012.
56. Julien S, Adriaenssens E, Ottenberg K, Furlan A, Courtand G, Vercoutter-Edouart AS, Hanisch FG, Delannoy $P$ and Le Bourhis X: ST6GalNAc I expression in MDA-MB-231 breast cancer cells greatly modifies their $\mathrm{O}$-glycosylation pattern and enhances their tumourigenicity. Glycobiology 16: 54-64, 2006.

57. Clément M, Rocher J, Loirand G and Le Pendu J: Expression of sialyl-Tn epitopes on betal integrin alters epithelial cell phenotype, proliferation and haptotaxis. J Cell Sci 117: 5059-5069, 2004.

58. Chou HH, Takematsu H, Diaz S, Iber J, Nickerson E, Wright KL, Muchmore EA, Nelson DL, Warren ST and Varki A: A mutation in human CMP-sialic acid hydroxylase occurred after the Homo-Pan divergence. Proc Natl Acad Sci USA 95: 11751-11756, 1998.

59. Hayakawa T, Satta Y, Gagneux P, Varki A and Takahata N: Alu-mediatedinactivation of the humanCMP-N-acetylneuraminic acid hydroxylase gene. Proc Natl Acad Sci USA 98: 11399-11404, 2001.

60. Bardor M, Nguyen DH, Diaz S and Varki A: Mechanism of uptake and incorporation of the non-human sialic acid $\mathrm{N}$-glycolylneuraminic acid into human cells. J Biol Chem 280: 4228-4237, 2005.

61. Yin J,Hashimoto A,Izawa M,Miyazaki K, Chen GY,Takematsu H, Kozutsumi Y, Suzuki A, Furuhata K, Cheng FL, et al: Hypoxic culture induces expression of sialin, a sialic acid transporter, and cancer-associated gangliosides containing non-human sialic acid on human cancer cells. Cancer Res 66: 2937-2945, 2006.

62. Alfonso S, Valdés-Zayas A, Santiesteban ER, Flores YI, Areces F, Hernández M, Viada CE, Mendoza IC, Guerra PP, García E, et al: A randomized, multicenter, placebo-controlled clinical trial of racotumomab-alum vaccine as switch maintenance therapy in advanced non-small cell lung cancer patients. Clin Cancer Res 20: 3660-3671, 2014

63. Cacciavillano W, Sampor C, Venier C, Gabri MR, de Dávila MT, Galluzzo ML, Guthmann MD, Fainboim L, Alonso DF and Chantada GL: A phase I study of the anti-idiotype vaccine racotumomab in neuroblastoma and other pediatric refractory malignancies. Pediatr Blood Cancer 62: 2120-2124, 2015.

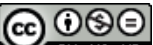

This work is licensed under a Creative Commons Attribution-NonCommercial-NoDerivatives 4.0 International (CC BY-NC-ND 4.0) License. 